\title{
Revised neutrino-gallium cross section and prospects of BEST in resolving the gallium anomaly
}

\author{
Vladislav Barinov, ${ }^{1,2, *}$ Bruce Cleveland, ${ }^{3, \dagger}$ Vladimir Gavrin, ${ }^{1, *}$ Dmitry Gorbunov, ${ }^{1,4,}$ and Tatiana Ibragimova ${ }^{1, \|}$ \\ ${ }^{1}$ Institute for Nuclear Research of the Russian Academy of Sciences, Moscow 117312, Russia \\ ${ }^{2}$ Physics Department, Moscow State University, Vorobievy Gory, Moscow 119991, Russia \\ ${ }^{3}$ SNOLAB, 1039 Regional Road 24 Lively Ontario, P3Y 1N2, Canada \\ ${ }^{4}$ Moscow Institute of Physics and Technology, Dolgoprudny 141700, Russia
}

(Received 24 October 2017; published 3 April 2018)

\begin{abstract}
$\mathcal{O}(1) \mathrm{eV}$ sterile neutrino can be responsible for a number of anomalous results of neutrino oscillation experiments. This hypothesis may be tested at short base line neutrino oscillation experiments, several of which are either ongoing or under construction. Here, we concentrate on the so-called gallium anomaly, found by SAGE and GALLEX experiments, and its foreseeable future tests with BEST experiment at Baksan Neutrino Observatory. We start with a revision of the neutrino-gallium cross section that is performed by utilizing the recent measurements of the nuclear final state spectra. We accordingly correct the parameters of gallium anomaly and refine the BEST prospects in testing it and searching for sterile neutrinos. We further evolve the previously proposed idea to investigate the anomaly with ${ }^{65} \mathrm{Zn}$ artificial neutrino source as a next option available at BEST and estimate its sensitivity to the sterile neutrino model parameters following the Bayesian approach. We show that after the two stages of operation BEST will make $5 \sigma$ discovery of the sterile neutrinos, if they are behind the gallium anomaly.
\end{abstract}

DOI: 10.1103/PhysRevD.97.073001

\section{INTRODUCTION}

Sterile neutrinos are hypothetical massive Majorana fermions, singlets with respect to the Standard Model (SM) gauge group, which have been introduced to explain oscillations of the SM (or active) neutrinos via mixing with them. There are no direct evidences for the sterile neutrinos, unless one interprets the results of the (anti)neutrino oscillation experiments, missing a fit by three active neutrinos, as observations of $\mathcal{O}(1) \mathrm{eV}$ sterile neutrinos. Though rather speculative, this interpretation encourages physicists to put forward various experimental proposals [1] to check this hypothesis and hunt the sterile neutrinos. One of such proposals, the Baksan experiment on sterile transitions (BEST) [1-5] is a short base line oscillation experiment aimed at searches/measurements of the disappearance of an electron neutrino by capturing it on gallium,

\footnotetext{
*barinov.vvl@gmail.com

bclevela@snolab.ca

gavrin@inr.ru

gorby@ms2.inr.ac.ru

tvi@inr.ru
}

Published by the American Physical Society under the terms of the Creative Commons Attribution 4.0 International license. Further distribution of this work must maintain attribution to the author(s) and the published article's title, journal citation, and DOI. Funded by SCOAP ${ }^{3}$.

$$
\nu_{e}+{ }^{71} \mathrm{Ga} \rightarrow \mathrm{e}^{-}+{ }^{71} \mathrm{Ge} .
$$

Neutrinos come from an artificial source, which is supposed to be ${ }^{51} \mathrm{Cr}$. It provides with direct testing of the gallium anomaly [6-8], which includes four measurements in total, and three out of four was performed with ${ }^{51} \mathrm{Cr}$ sources.

In this paper, we study the recently proposed idea [5] to perform after a ${ }^{51} \mathrm{Cr}$-based experiment, the second stage of the BEST operation with the neutrino source based on the isotope ${ }^{65} \mathrm{Zn}$. The main advantage of ${ }^{65} \mathrm{Zn}$ with respect to ${ }^{51} \mathrm{Cr}$ is higher availability. At the same time, neutrino spectra of ${ }^{51} \mathrm{Cr}$ and ${ }^{65} \mathrm{Zn}$ are significantly different which allows us to achieve a more "uniform" sensitivity to the sterile neutrino parameter space after the BEST subsequent operation with the two artificial sources. In this case, to estimate the BEST sensitivity to sterile neutrino parameters, we calculate the cross section of the process (1) at neutrino energies expected for the ${ }^{65} \mathrm{Zn}$ source. To this end, we use the computer program SPECCROS by John Bahcall, which we adapt to account for recent measurements of Refs. $[9,10]$. We revise the estimates of the cross section of the process (1) utilized by SAGE and GALLEX, and consequently, refine the parameters of sterile neutrino model favored by the gallium anomaly $[4,11,12]$. Then, we reestimate the prospects of testing the gallium anomaly at BEST with an artificial source based on a ${ }^{51} \mathrm{Cr}$ isotope. 
Finally, we find the sensitivity of BEST with a ${ }^{65} \mathrm{Zn}$ source to the sterile neutrino model parameters. In accordance with expectation, we observe that running the subsequent experiments with the ${ }^{51} \mathrm{Cr}$ and ${ }^{65} \mathrm{Zn}$ neutrino sources considerably improves the BEST performance. In particular, it would allow us to make a $5 \sigma$ discovery and determine the sterile neutrino model parameters with $10 \%$ accuracy.

The paper is organized in the following way. The neutrino-gallium cross section is revisited in Sec. II. In particular, here we obtain formulas valid for ${ }^{37} \mathrm{Ar},{ }^{51} \mathrm{Cr}$, and ${ }^{65} \mathrm{Zn}$ sources. Section III contains a sketch of BEST. In Sec. IV we apply the results, obtained in previous sections, to refine the gallium anomaly, revise the BEST sensitivity with a ${ }^{51} \mathrm{Cr}$ source, and investigate BEST perspectives with a ${ }^{65} \mathrm{Zn}$ source in testing the gallium anomaly and searches for sterile neutrinos. We summarize in Sec. V.

\section{NEUTRINO-GALLIUM CROSS SECTION}

The general formula for neutrino absorption cross section accounting for nuclear transitions in reaction (1) can be cast in the following form [13]:

$$
\sigma=\sigma_{0}\left\langle\omega_{e}^{2} G\left(Z, \omega_{e}\right)\right\rangle,
$$

where the expression in brackets is the dimensionless phase space factor and $\sigma_{0}$ refers to the scale of the neutrino capture cross section.

The scale factor $\sigma_{0}$ can be written as $[14,15]$

$$
\sigma_{0}=\frac{4 \pi^{3} \log 2 \alpha \hbar^{3}}{m_{e}^{3} c^{4}}\left(\frac{2 J_{f}+1}{2 J_{i}+1}\right) \frac{Z}{f t_{1 / 2}\left({ }^{71} \mathrm{Ge}\right)},
$$

where $\alpha$ is the fine structure constant, $J_{f}$ is spin of the final nuclear state, $J_{i}$ is spin of the initial nuclear state, $Z$ is atomic number of the final nucleus, $f t_{1 / 2}\left({ }^{71} \mathrm{Ge}\right)$ is the product of dimensionless phase-space factor $f$ for the kinematically allowed electron capture, the inverse process to the reaction (1), and $t_{1 / 2}\left({ }^{71} \mathrm{Ge}\right)$ is the half-life of ${ }^{71} \mathrm{Ge}$. This factor is defined as

$f t_{1 / 2}\left({ }^{71} \mathrm{Ge}\right) \equiv \frac{2 \pi^{3} \log 2 \hbar^{7}}{m_{e}^{5} c^{4}} \frac{1}{\left(G_{V}^{2}\left|M_{i, f}\right|_{F}^{2}+G_{A}^{2}\left|M_{i, f}\right|_{G T}^{2}\right)}$,

where $G_{V}, G_{A}$ are the vector and axial coupling constants of nucleon, determined from the neutron decay [16], and $\left|M_{i, f}\right|_{F}^{2},\left|M_{i, f}\right|_{G T}^{2}$ are the squares of the transition matrix elements, which the vector current (Fermi transitions) and the axial-vector current (Gamow-Teller transitions) contribute to $[17,18]$. These allowed transitions are illustrated in Table I, and the squared transition matrix elements read [17],
TABLE I. Types of allowed transitions. $\mathrm{S}$ is total spin of the leptons. $\Delta \mathrm{L}$ is the change of the total angular momentum of the system. $\Delta \mathrm{P}$ is the change of parity of the system.

\begin{tabular}{ll}
\hline \hline Fermi transitions & Gamow-Teller transitions \\
\hline$\frac{1}{2} \uparrow_{n} \rightarrow \frac{1}{2} \uparrow_{p}+\frac{1}{2} \uparrow_{e}+\frac{1}{2} \downarrow_{\nu}$ & $\frac{1}{2} \uparrow_{n} \rightarrow \frac{1}{2} \downarrow_{p}+\frac{1}{2} \uparrow_{e}+\frac{1}{2} \uparrow_{\nu}$ \\
$\mathrm{S}=0, \Delta \mathrm{L}=0, \Delta \mathrm{P}=0$ & $\mathrm{~S}=1, \Delta \mathrm{L}= \pm 1, \Delta \mathrm{P}=0$ \\
\hline \hline
\end{tabular}

$$
\begin{gathered}
\left|M_{i, f}\right|_{F}^{2}=\left|\left\langle f\left|\sum_{n=1}^{A} Q_{n}^{+}\right| i\right\rangle\right|^{2}, \\
\left|M_{i, f}\right|_{G T}^{2}=\sum_{j=-1,0,1}\left|\left\langle f|| \sum_{n=1}^{A} Q_{n}^{+} \sigma_{j} \mid i\right\rangle\right|^{2},
\end{gathered}
$$

where $Q_{n}^{+}$is the transformation operator of neutron into proton without a spin flip, and the sum is taken over all nucleons in the nucleus; $2 \times 2$ spin matrices $\sigma_{j}$ are related to the Pauli matrices $\tau_{i}$ as follows:

$\sigma_{1}=\frac{1}{\sqrt{2}}\left(\tau_{1}+i \tau_{2}\right), \quad \sigma_{0}=\tau_{3}, \quad \sigma_{-1}=\frac{1}{\sqrt{2}}\left(\tau_{1}-i \tau_{2}\right)$.

Summations in (5), (6) go over the spin matrices for all possible orientations of the angular momentum of the nucleon in the final state, since the transition probability (due to invariance with respect to rotations) should not depend on the magnetic quantum number of the initial state.

Following the works of John Bahcall $[13,14,19]$, we introduced in (2) the value of $\left\langle\omega_{e}^{2} G\left(Z, \omega_{e}\right)\right\rangle$, where $G\left(Z, \omega_{e}\right) \equiv p_{e} F\left(Z, \omega_{e}\right) / 2 \pi \alpha Z \omega_{e}$, is the dimensionless phase-space factor averaged over the electron energies. The explicit expression is given by the formula

$$
\left\langle\omega_{e}^{2} G\left(Z, \omega_{e}\right)\right\rangle \equiv \frac{\int_{\omega_{e}^{\min }}^{\omega_{\max }^{\max }} \omega_{e} p_{e} F\left(Z, \omega_{e}\right) \phi\left(q_{\nu}\right) d \omega_{e}}{2 \pi \alpha Z \int_{0}^{q_{\nu}^{\max }} \phi\left(q_{\nu}\right) d q_{\nu}},
$$

where $\phi\left(q_{\nu}\right)$ is the neutrino energy distribution function, $q_{\nu}=E_{\nu} / m_{e} c^{2}$ is the dimensionless neutrino energy, $\omega_{e} \equiv E / m_{e} c^{2}, p_{e}=p / m_{e} c$ are the dimensionless energy and momentum of the electron. The integrals in (8) are taken over the whole spectrum of electrons, which energy can be expressed as

$$
\begin{aligned}
E= & E_{\nu}+[M(A, Z-1)-M(A, Z)] c^{2} \\
& +m_{e} c^{2}-\left\langle E_{e x}\right\rangle-V_{0},
\end{aligned}
$$

where $E_{\nu}$ is the energy of the incoming neutrino, $\left\langle E_{e x}\right\rangle$ is the average excitation energy of the produced nucleus, $V_{0}$ is a correction [20] for the smaller average binding energy of electron inside the nucleus with respect to that outside, and the term in parenthesis is the atomic mass difference between the initial $M(A, Z-1)$ and final $M(A, Z)$ atomic masses. 
Quantity $F\left(Z, \omega_{e}\right)$, which enters into formula (8), accounts for the nonplane wave structure of the electron wave function and is closely related to the Fermi function [21], that is the ratio of electron squared wave functions calculated with and without the Coulomb potential,

$F(Z, E, r)=2\left(1+\gamma_{0}\right)(2 p r / \hbar)^{2\left(\gamma_{0}-1\right)} e^{\pi \nu} \frac{\left|\Gamma\left(\gamma_{0}+i \nu\right)\right|^{2}}{\left[\Gamma\left(2 \gamma_{0}+1\right)\right]^{2}}$.

Here, we introduced $\gamma_{0} \equiv\left[1-(\alpha Z)^{2}\right]^{1 / 2}, \nu \equiv \alpha Z E / p_{e} c$, and $r$ denotes distance from the nucleus center to the electron. According to [14], the expression (10) must be averaged over the entire finite volume $V$ of the nucleus of a radius $R$, that reveals

$$
\begin{aligned}
F\left(Z, \omega_{e}\right) & =\frac{1}{V} \int_{0}^{R} F\left(Z, \omega_{e}, r\right) d V \\
& =\left[\frac{3}{2 \gamma_{0}+1}\right] F\left(Z, \omega_{e} ; r=R\right) .
\end{aligned}
$$

The resulting correction reflects the fact that electron capture can occur at any point inside the nucleus. There are also corrections [20] to $F\left(Z, \omega_{e}\right)$ due to the shielding of the Coulomb potential inside the nucleus. We take them into account, but find them small, at the level of the percent for the set of interesting neutrino energies.

The review presented above in this section concerns only the allowed nuclear transitions. The question of the contribution of the excited states of the nucleus to the total neutrino absorption cross section is discussed below.

In a paper [22], Hata and Haxton have shown that the contribution of excited states to the total neutrino absorption cross section on ${ }^{71} \mathrm{Ga}$ can be written as

$$
\sigma=\sigma_{\text {g.s. }}\left[1+\frac{\sum_{E_{x}} \lambda_{E_{x}} B(G T)_{E_{x}}}{B(G T)_{\text {g.s. }}}\right] .
$$

Here, $\sigma_{\text {g.s. }}$ is the neutrino absorption cross section associated with the gallium ${ }^{71} \mathrm{Ga}$ transition to the ground state of germanium ${ }^{71} \mathrm{Ge}$, which is given by Eq. (2), the coefficients $\lambda_{E_{x}}$ are the phase space factors for these transitions normalized to the ground-state phase space factor [12]. These coefficients can be calculated from Eq. (8) by making use of the program SPECCROS written by John Bahcall, $B(G T)_{\text {g.s. }}$ is the square of the Gamow-Teller transition matrix element to the ground state (see Table I), and $B(G T)_{E_{x}}$ are the squared matrix elements responsible for transitions to excited states with energies $E_{x}$ [23] measured from the ground state.

The gallium decay scheme, depicted in Fig. 1, presents transitions to excited states with excitation energies $E_{x}$ of 175 and $500 \mathrm{keV}$, relevant for artificial sources of neutrinos based on the radioactive isotopes ${ }^{51} \mathrm{Cr}$ [6] and ${ }^{37} \mathrm{Ar}$ [7].
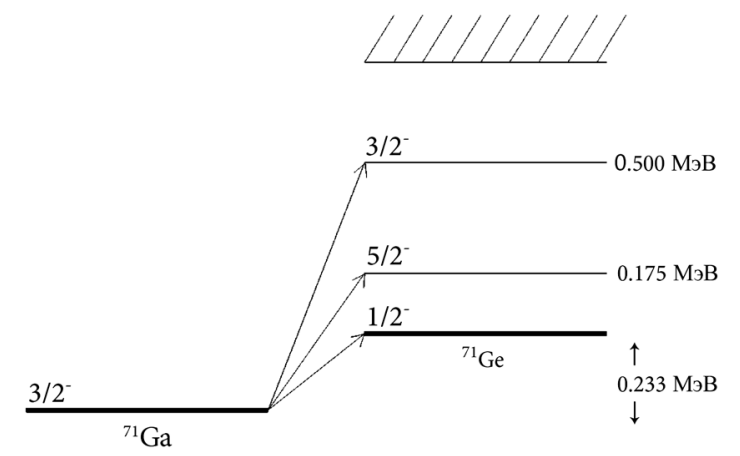

FIG. 1. Scheme of the ${ }^{71} \mathrm{Ga} \rightarrow{ }^{71} \mathrm{Ge}$ transitions induced by electron neutrinos emitted in weak decays of ${ }^{51} \mathrm{Cr}$ and ${ }^{37} \mathrm{Ar}$.

However, for an artificial neutrino source ${ }^{65} \mathrm{Zn}$ [24], the higher energy levels get excited in the process (1), and their contribution to the total cross section is significant, $\sim 20 \%-30 \%$. The coefficients $\lambda_{E_{x}}$ for these transitions for ${ }^{65} \mathrm{Zn}$ are $\lambda_{175}=0.7969, \quad \lambda_{500}=0.4791, \lambda_{708}=0.3145$, $\lambda_{808}=0.2466$, and $\lambda_{1096}=0.0934$; the squared transition matrix elements $B(G T)_{E_{x}}$ corresponding to these energies are given in [23].

Based on the new results of measuring the threshold energy of the gallium transition to the ground state of germanium [10],

$$
Q=233.5 \pm 1.2 \mathrm{keV},
$$

and using the half-life of ${ }^{71} \mathrm{Ge}\left(t_{1 / 2}\left({ }^{71} \mathrm{Ge}\right)=11.43 \pm 0.03 \mathrm{~d}\right)$ [25], we calculate $\log f t_{1 / 2}$ using the $f t$ calculator [26],

$$
\log f t_{1 / 2}\left({ }^{71} \mathrm{Ge}\right)=4.353 \pm 0.005 .
$$

We exploit (14) further to calculate the Gamow-Teller transition matrix element $B(G T)_{\text {g.s. }}$, which can be written as [12,14], cf. (4),

$B(G T)_{\text {g.s. }}=\left[\frac{2 J_{f}+1}{2 J_{i}+1}\right] \frac{2 \pi^{3} \log 2 \hbar^{7}}{G_{F}^{2}\left|V_{u d}\right|^{2} m_{e}^{5} c^{4} g_{A}^{2} f t_{1 / 2}\left({ }^{71} \mathrm{Ge}\right)}$,

where $G_{F}$ is the Fermi constant, $V_{u d}$ is the element of the Cabibbo-Kobayashi-Maskawa mixing matrix [16], $g_{A}=$ $-1.2723(23)$ is the axial coupling constant [16], and $G_{A}^{2}=G_{F}^{2}\left|V_{u d}\right|^{2} g_{A}^{2}$. Numerically, one finds

$$
B(G T)_{\text {g.s. }}=0.086 \pm 0.001,
$$

while from Eqs. (3) and (14),

$$
\sigma_{0}=(8.6 \pm 0.1) \times 10^{-46} \mathrm{~cm}^{2}
$$

While the central value of (17) is fully consistent with previous estimate [13], 
TABLE II. Neutrino energy spectra-energies $E_{\nu}$ and neutrino fractions $f_{E_{\nu}}$-and corresponding neutrino capture cross section on gallium for the set of artificial sources under consideration.

\begin{tabular}{lcrc}
\hline \hline Isotope & $E_{\nu}, \mathrm{MeV}$ & \multicolumn{1}{c}{$f_{E_{\nu}}, \%$} & $\sigma\left(E_{\nu}\right), 10^{-46} \mathrm{~cm}^{2}$ \\
\hline${ }^{51} \mathrm{Cr}$ & & & \\
& 0.752 & $8.49(1)$ & $63.22 \pm 1.40$ \\
& 0.747 & $81.63(1)$ & $62.58 \pm 1.39$ \\
& 0.432 & $0.93(1)$ & $27.14 \pm 0.52$ \\
${ }^{37} \mathrm{Ar}$ & 0.427 & $8.95(1)$ & $26.72 \pm 0.51$ \\
& & & \\
& 0.813 & $9.80(1)$ & $71.63 \pm 1.62$ \\
${ }^{65} \mathrm{Zn}$ & 0.811 & $90.20(1)$ & $71.35 \pm 1.61$ \\
& & & \\
\hline \hline
\end{tabular}

$$
\sigma_{0}^{\text {Bahcall }}=(8.611 \pm 0.011) \times 10^{-46} \mathrm{~cm}^{2},
$$

the uncertainty saturated by that of (13) is significantly larger. It happened because the value (17) was obtained from an analysis of the new data [10]. We utilize the new estimate of the threshold energy of the gallium transition to the ground state of germanium (13), in contrast to the old value $Q=232.69 \pm 0.15 \mathrm{keV}$ used previously in [13]. We use the most recent value (13) and hence (16), which are consistent with previous results, while their errors do not dominate the uncertainties of our estimates of the neutrinocapture cross sections.

Further, for each spectral line of the artificial sources ${ }^{51} \mathrm{Cr},{ }^{37} \mathrm{Ar}$, and ${ }^{65} \mathrm{Zn}$ presented ${ }^{1}$ in Table II, the values of $\sigma_{\text {g.s. }}$ and $\lambda_{E_{x}}$ entering (12) are calculated from (2) and data $[10,23]$ by making use of the program SPECCROS. Subsequently, for each neutrino energy, the neutrino capture cross section is obtained including contributions of the kinematically allowed excited states, see Table II. Then the total neutrino absorption cross sections for each artificial source are obtained by summing over all energies weighted with the corresponding relative fractions,

$$
\sigma=\sum_{E_{\nu}} \sigma\left(E_{\nu}\right) f_{E_{\nu}} .
$$

The results are as follows:

$$
\begin{gathered}
\sigma\left({ }^{51} \mathrm{Cr}\right)=(59.10 \pm 1.14) \times 10^{-46} \mathrm{~cm}^{2}, \\
\sigma\left({ }^{37} \mathrm{Ar}\right)=(71.38 \pm 1.46) \times 10^{-46} \mathrm{~cm}^{2}, \\
\sigma\left({ }^{65} \mathrm{Zn}\right)=(87.76 \pm 2.03) \times 10^{-46} \mathrm{~cm}^{2} .
\end{gathered}
$$

We use these estimates in the following sections.

\footnotetext{
${ }^{1}$ The lowest line of ${ }^{65} \mathrm{Zn}$ with close to the threshold energy (13) $E=0.236 \mathrm{keV}$ is kinematically forbidden to produce an electron [after an account of a somewhat lower electromagnetic binding energy $V_{0}$ of an electron inside the nucleus with respect to that outside, see Eq. (9)].
}

\section{SKETCH OF BEST}

The BEST experiment is described in detail in Ref. [4]. Here, we merely recall the general idea of this experiment.

The experimental setup consists of two concentric zones filled with liquid gallium. The first zone is a sphere of radius $R_{1}=0.66 \mathrm{~m}$, in the center of which there is an artificial neutrino source about $0.1 \mathrm{~m}$ in size. Such a size makes it possible to place in the center of the first zone a source of neutrinos ${ }^{51} \mathrm{Cr}$ with an activity $3 \mathrm{MCi}$. The second zone is a cylinder of radius $R_{2}=1.096 \mathrm{~m}$ and height $2 \times R_{2}$. The image of the experimental setup is shown in Fig. 2. The liquid gallium is irradiated by a neutrino flux from an artificial source. As a result of the reaction (1), germanium atoms are formed, which are then chemically extracted from the zones. Possible transitions to sterile neutrinos would affect the neutrino flux. Hence, the numbers of extracted atoms are sensitive to the presence of light sterile neutrinos.

The total mass of gallium is 50 tons. The original proposal $[1,2]$ suggests to exploit the isotope ${ }^{51} \mathrm{Cr}$ as the artificial neutrino source with a radioactivity of about $3 \mathrm{MCi}$. At the same time, other candidates may be considered, and one of the most promising is ${ }^{65} \mathrm{Zn}$ [5]. It provides a different neutrino spectrum giving the opportunity to test a somewhat different region of sterile neutrino parameter space. Also the half-life of ${ }^{65} \mathrm{Zn}$ is longer ( $244 \mathrm{~d}$ compared to $27 \mathrm{~d}$ for ${ }^{51} \mathrm{Cr}$ ), thus giving more time to make longer measurements with the sufficient activity of the source. However, the artificial source ${ }^{65} \mathrm{Zn}$ of the same activity has a noticeably larger size than the source ${ }^{51} \mathrm{Cr}$, which reduces the oscillation signal after averaging over the source volume. This must be avoided, and a special investigation is required to find the reliable technical

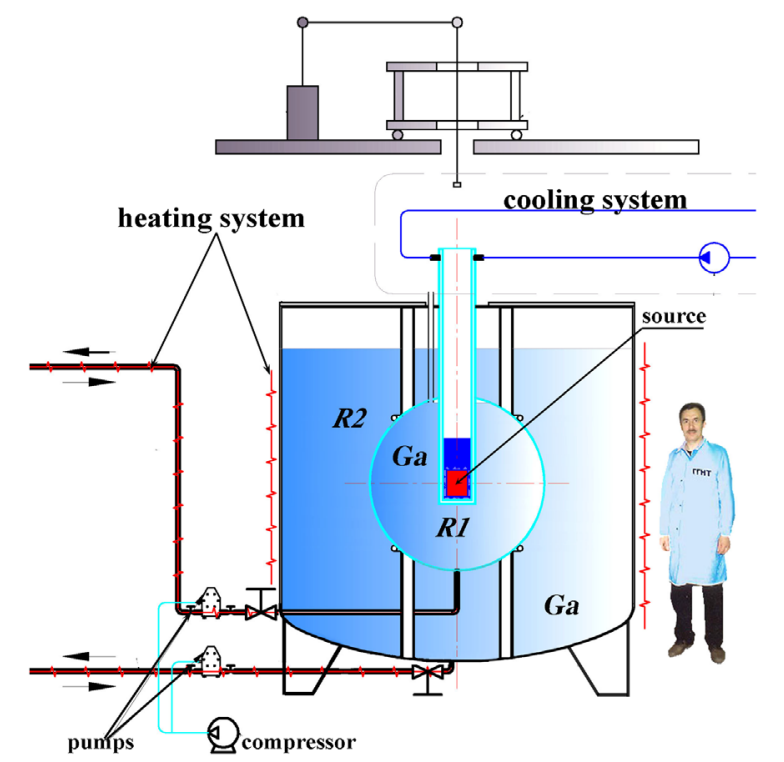

FIG. 2. The BEST layout; vessel sizes are $R_{1}=0.66 \mathrm{~m}$, $R_{2}=1.096 \mathrm{~m}$. 
solution and optimize the source volume. For the present study, we take as a realistic option to adopt the smaller ${ }^{65} \mathrm{Zn}$ source with an activity of about $1 \mathrm{MCi}$, which will be acceptably compact. The volume occupied by the source within the first zone will increase slightly, but this will not negatively affect the isotropy of the target irradiation. Likewise, with such activity, it will be possible to keep a sufficiently high homogeneity of the zinc source. Finally, the lower power of the source is partly compensated by a larger cross section (21). Although the predicted production rate from the ${ }^{65} \mathrm{Zn}$ source with an activity of about $1 \mathrm{MCi}$ is about 2 times smaller compared to the $3 \mathrm{MCi}{ }^{51} \mathrm{Cr}$ source, nevertheless, the expected number of germanium atoms to be extracted from the vessels are still sufficiently large with respect to the solar background. The statistical errors grow insufficiently, and the total uncertainty of the extraction is dominated by systematics, which we expect to be the same as in case of the ${ }^{51} \mathrm{Cr}$ source.

\section{REVISION OF THE GALLIUM ANOMALY AND SEARCHES AT BEST}

For the revision of the results for neutrino absorption cross sections, we begin with discussion of uncertainties.

The main contribution to the uncertainty of the neutrino absorption cross section is associated with corrections from the excited states. To calculate the uncertainty of a neutrino cross section, the results of [10,23], as well as the known uncertainty of $\sigma_{0}$ are accounted for. Assuming the measurements of $B(G T)$ for different energy levels to be independent, we calculate the overall error for each spectral line of the artificial sources as the square root of the sum of the squared standard deviations of all values entering (12).

The obtained values of the cross sections for ${ }^{51} \mathrm{Cr}$ and ${ }^{37} \mathrm{Ar}$ and their relative uncertainties deviate insignificantly from the previous study in [12]. However, we take a different value of the energy of the gallium transition to the ground state of germanium [10], as well as another value of the transition matrix element to the ground state (16). We find the uncertainty of the cross sections to be about $2 \%$, while earlier for the BEST experiment, the uncertainty of $+3.6 \% /-2.8 \%$ [13] has been adopted.

It is worth noting that the measurement of the threshold energy of the gallium-germanium transition does not contain unknown uncertainties in the nuclear structure, which could explain the anomalous results of the SAGE [6,7] and GALLEX [8] experiments. This result was further discussed in Ref. [10].

The results obtained in Sec. II imply that despite the fact that we applied a new value of the threshold energy of the gallium transition into the ground state of germanium than previously done, and despite the utilization of the recent measurements of the transitions matrix elements [23], the central values and their uncertainties have not changed much, in comparison with the values presented
TABLE III. Values of the magnitudes of the gallium anomaly, obtained on the basis of the refined data on the neutrino absorption cross section, using the value of $Q=233.5 \pm$ $1.2 \mathrm{keV}$, the transition matrix element to the ground state $B G T_{\text {g.s. }}=0.086 \pm 0.001$, and the transition matrix elements to excited states taken from Ref. [23].

\begin{tabular}{cccccc}
\hline \hline & SAGE 1 & SAGE 2 & GALLEX 1 & GALLEX 2 & AVE \\
\hline$R$ & $0.93_{-0.12}^{+0.12}$ & $0.77_{-0.08}^{+0.09}$ & $0.93_{-0.11}^{+0.11}$ & $0.80_{-0.11}^{+0.11}$ & $0.84_{-0.05}^{+0.05}$ \\
\hline \hline
\end{tabular}

in [12]. The refined values of the ratios of the observed-toexpected number of events $R$ in gallium experiments (gallium anomaly), which we represent in this paper, see Table III, almost completely coincide with the values presented in [12].

Thus, taking into account the refined value of the neutrino absorption cross section on gallium found in this paper, the resulting error of the experiment BEST [3] for the source ${ }^{51} \mathrm{Cr}$ is $4.9 \%$ for each of the zones and $4.2 \%$ for the total target, instead of $5.5 \%$ and $4.8 \%$, respectively. For the artificial neutrino source ${ }^{65} \mathrm{Zn}$ with an activity of $1 \mathrm{MCi}$ in the BEST experiment, the resulting errors will be the same as for the $3 \mathrm{MCi}^{51} \mathrm{Cr}$ source if the irradiation plan with the ${ }^{65} \mathrm{Zn}$ source is identical to that presented in Ref. [3].

The anomalous lack of neutrinos presented in Table III can be explained by oscillations of electron neutrinos into sterile partners [27]. The combined results of SAGE and GALLEX, obtained on the basis of refined data, are presented in Fig. 3. The result shown in Fig. 3 shows that the best fit values $\Delta m^{2}=2.5 \mathrm{eV}^{2}$ and $\sin ^{2}(2 \vartheta)=0.3$ are slightly different (by about 10\%) from those presented in [4]. The refined regions of the neutrino oscillation

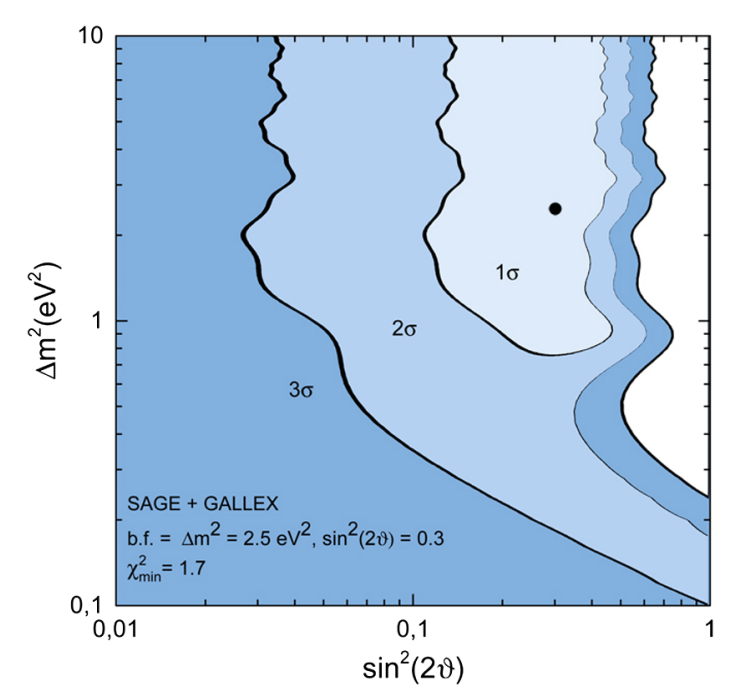

FIG. 3. Allowed regions of oscillation parameters obtained by combining the results of SAGE + GALLEX using the refined data presented above. 


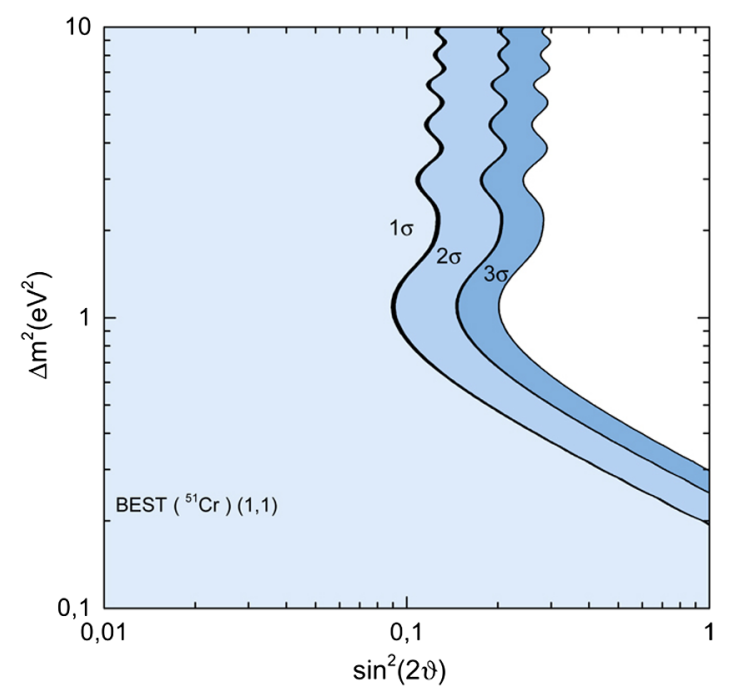

FIG. 4. Allowed regions of oscillation parameters in case the BEST experiment does not find any anomalies: the ratios $R$ of the observed-to-expected without sterile neutrinos germanium atoms in both vessels are consistent with unity, $(1,1)$.

parameters to be tested at the BEST [3] experiment with the artificial source ${ }^{51} \mathrm{Cr}$ are given in Figs. 4 and 5. They are found by applying the formulas from [4]. Assuming the BEST with the source ${ }^{51} \mathrm{Cr}$ fully confirms the anomaly, the most favorable regions (all data of the three experiments are included) of the sterile neutrino model parameter space are presented in Fig. 6. Comparing these plots with similar ones in Ref. [4], one can conclude that after revision of the neutrino capture cross section, all signal regions become more compact; hence, the sensitivity of BEST to the sterile neutrino model certainly increases.

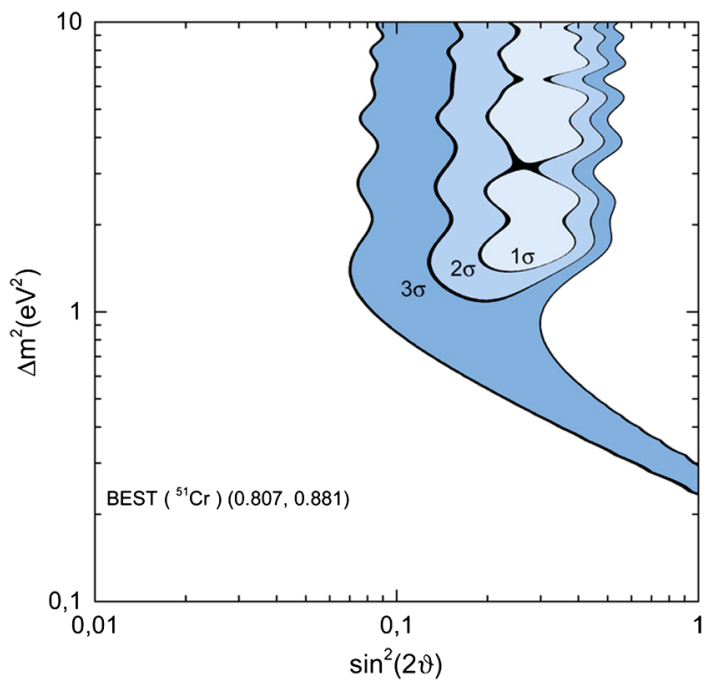

FIG. 5. Allowed regions of oscillations parameters if the result of the BEST experiment corresponds to the best fit point for combining the SAGE + GALLEX. The numbers in parentheses indicate the most probable ratios $R$ of the observed-to-expected without sterile neutrinos germanium atoms in the two vessels.

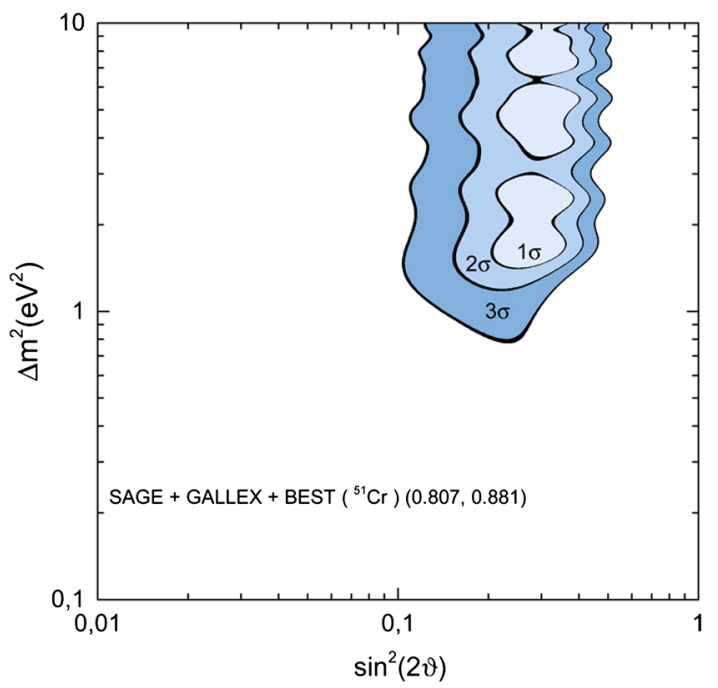

FIG. 6. Allowed regions of oscillation parameters, when the results of SAGE + GALLEX are combined with the result of BEST for ${ }^{51} \mathrm{Cr}$ source consistent with the SAGE + GALLEX best fit point.

To illustrate the power of the source ${ }^{65} \mathrm{Zn}$ in further testing the sterile neutrino hypothesis, we present in Fig. 7, the anomaly favored region after the second run of BEST operating with the source ${ }^{65} \mathrm{Zn}$. The sensitivity of the second run is estimated in exactly the same way as has been done in [4] for the source ${ }^{51} \mathrm{Cr}$. For the favored by gallium anomaly best fit values of the sterile neutrino model, the expected signal rates in the two vessels of BEST correspond to ratios $R=(0.827,0.781)$. One clearly observes from Figs. 6 and 7 the significant improvement in the sensitivity after the combined analysis of the two runs

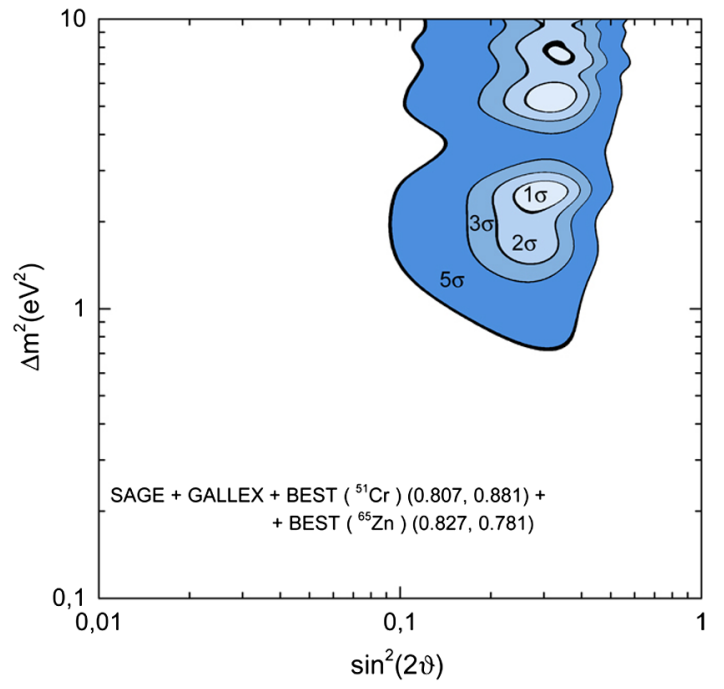

FIG. 7. Allowed regions of oscillation parameters, built on the basis of new data, in the case of combining the results of SAGE + GALLEX with the result of BEST for two sources $\left({ }^{51} \mathrm{Cr}\right.$ and ${ }^{65} \mathrm{Zn}$ ), which corresponds to the best fit point. 


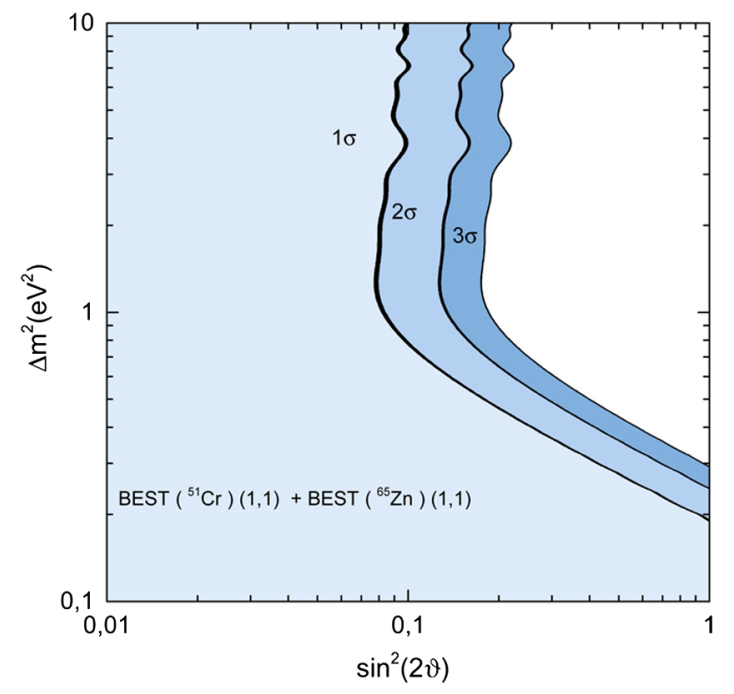

FIG. 8. Allowed regions of oscillation parameters in case the BEST experiment does not find any anomalies after two runs: the ratios $R$ of the observed-to-expected without sterile neutrinos germanium atoms in both vessels for both sources are consistent with unity, $(1,1)$.

(assuming both confirm the gallium anomaly). Finally, if both runs find no hint of sterile neutrinos, the exclusion region will expand with respect to that in Fig. 4, and it is presented in Fig. 8.

\section{SUMMARY}

In this work, updated data $[10,23]$ on neutrino absorption cross section on gallium and the program SPECCROS are used to refine the neutrino absorption cross section, which is done for ${ }^{71} \mathrm{Ga}$ and the neutrino sources ${ }^{51} \mathrm{Cr},{ }^{37} \mathrm{Ar}$, and ${ }^{65} \mathrm{Zn}$.

The results obtained for the sources ${ }^{51} \mathrm{Cr}$ and ${ }^{37} \mathrm{Ar}$ agree with the estimates presented in [12]. This suggests that the leading uncertainties in the cross section for neutrino capture are the uncertainties of the matrix elements of nuclear transitions to excited states. The analysis of the capture cross sections for all three types of neutrino sources considered in this paper reveals that taking into account all the uncertainties in the determination of the threshold energy of the gallium transition to the ground state of germanium and taking into account the uncertainties of the matrix elements of the transitions to excited states give an uncertainty of the cross sections of about $2 \%$. This result shows that the central values and errors of the cross sections (19)-(21) cannot explain the anomalous results

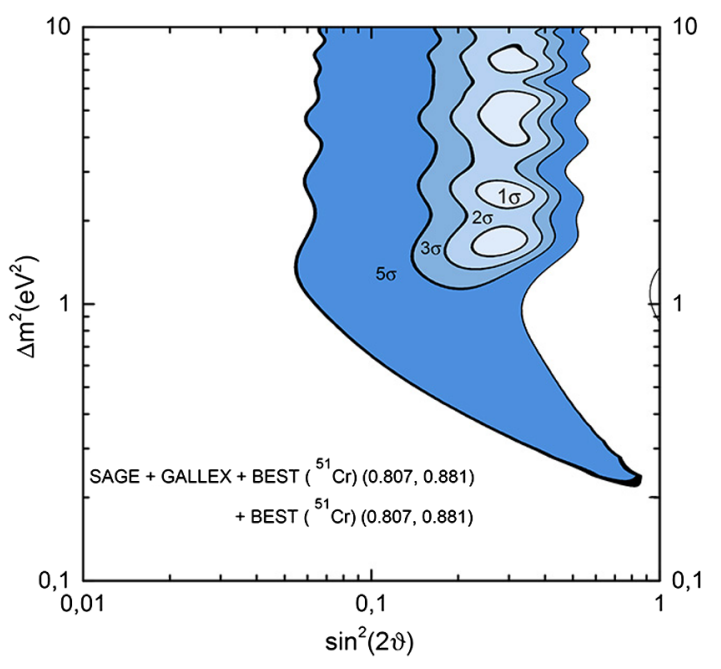

FIG. 9. Allowed regions of oscillation parameters in case the BEST experiment confirms the gallium anomaly in both runs performed with the chrome-51 sources.

of SAGE [6,7] and GALLEX [8]: the anomalous results remain intact.

Thus, the main results published in [4] where the data $[10,23]$ have not used, remain true, and the experiment BEST [3] has high potential in testing the hypothesis of electron neutrino oscillations into sterile neutrinos.

To summarize, we present the refined estimates of BEST sensitivity to models with light sterile neutrinos mixed with electron neutrinos. The obtained results strongly suggest to use the new artificial source based on the isotope ${ }^{65} \mathrm{Zn}$ at the second stage of BEST operation, which allow us to reduce the degeneracy in sensitivity to the sterile neutrino model parameters. To illustrate this point, we present in Fig. 9, the sensitivity contours in case of both stages exploiting the ${ }^{51} \mathrm{Cr}$ sources. One can conclude by comparing the plots in Figs. 7 and 9 that while the 5- $\sigma$ discovery of the sterile neutrinos is mostly due to double statistics (one stage is not enough to achieve this goal), the second source with different neutrino energies definitely provides with better cornering the signal regions with respect to the case of identical sources. We study possible impact of the future BEST results on the status of the gallium anomaly.

\section{ACKNOWLEDGMENTS}

We thank S. Kulagin, F. Simkovic, and O. Smirnov for valuable discussions. The work was supported by the RSF Grant No. 17-12-01547. 
[1] K. N. Abazajian et al., arXiv:1204.5379.

[2] V. N. Gavrin, V. V. Gorbachev, E. P. Veretenkin, and B. T. Cleveland, arXiv:1006.2103v2.

[3] V. Gavrin et al., Phys. Part. Nucl. 46, 131 (2015).

[4] V. Barinov, V. Gavrin, D. Gorbunov, and T. Ibragimova, Phys. Rev. D 93, 073002 (2016).

[5] V. N. Gavrin, B. T. Cleveland, V. V. Gorbachev, T. V. Ibragimova, A. V. Kalikhov, Y. P. Kozlova, Y. A. Malyshkin, I. N. Mirmov, A. A. Shikhin, and E. P. Veretenkin, J. Phys. Conf. Ser. 798, 012113 (2017).

[6] J. N. Abdurashitov et al. (SAGE Collaboration), Phys. Rev. C 59, 2246 (1999).

[7] J. N. Abdurashitov et al., Phys. Rev. C 73, 045805 (2006).

[8] F. Kaether, W. Hampel, G. Heusser, J. Kiko, and T. Kirsten, Phys. Lett. B 685, 47 (2010).

[9] D. Frekers et al., Phys. Lett. B 706, 134 (2011).

[10] D. Frekers et al., Phys. Lett. B 722, 233 (2013).

[11] C. Giunti and M. Laveder, Phys. Rev. C 83, 065504 (2011).

[12] C. Giunti, M. Laveder, Y. F. Li, Q. Y. Liu, and H. W. Long, Phys. Rev. D 86, 113014 (2012).

[13] J. N. Bahcall, Phys. Rev. C 56, 3391 (1997).
[14] J. N. Bachall, Neutrino Astrophysics (Cambridge University Press, Cambridge, England, 1989).

[15] K. Zuber, Neutrino Physics (CRC Press, London, 2012).

[16] C. Patrignani et al. (Particle Data Group), Chin. Phys. C 40, 100001 (2016).

[17] C.S. Wu and S. A. Moszkowski, Beta Decay (Wiley, New York, 1966).

[18] Z.-C. Gao, Y. Sun, and Y. S. Chen, Phys. Rev. C 74, 054303 (2006).

[19] J. N. Bahcall, Rev. Mod. Phys. 50, 881 (1978).

[20] J. N. Bahcall, Nucl. Phys. 75, 10 (1966).

[21] E. J. Konopinski, The Theory of Beta Radioactivity (Oxford University Press, New York, 1950).

[22] N. Hata and W. Haxton, Phys. Lett. B 353, 422 (1995).

[23] D. Frekers et al., Phys. Rev. C 91, 034608 (2015).

[24] Lne-lnhb, http://www.nucleide.org.

[25] W. Hampel and L. P. Remsberg, Phys. Rev. C 31, 666 (1985).

[26] National nuclear data center, http://www.nndc.bnl.gov/logft (2016).

[27] M. Laveder, Nucl. Phys. B, Proc. Suppl. 168, 344 (2007). 